\title{
Correction to: Association of Cannabis, Stimulant, and Alcohol use with Mortality Prognosis Among HIV-Infected Men
}

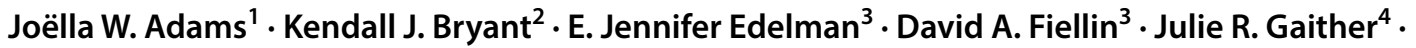 \\ Adam J. Gordon ${ }^{5,6} \cdot$ Kirsha S. Gordon $^{7} \cdot K_{\text {Kevin L. Kraemer }}^{8} \cdot$ Matthew J. Mimiaga $^{1}$ • Don Operario ${ }^{1}$ Janet P. Tate ${ }^{3,7}$. \\ Jacob J. van den Berg ${ }^{1} \cdot$ Amy C. Justice $^{3,7} \cdot$ Brandon D. L. Marshall $^{1}$
}

Published online: 8 March 2018

(c) Springer Science+Business Media, LLC, part of Springer Nature 2018

\section{Correction to: AIDS Behav https://doi.org/10.1007/s10461-017-1905-4}

In the original publication of the article, the given and family name of the third author was not correct. The name has been corrected with this erratum.

The original article can be found online at https://doi.org/10.1007/ s10461-017-1905-4.

\section{Brandon D. L. Marshall}

brandon_marshall@brown.edu

1 Brown University School of Public Health, Providence, RI, USA

2 National Institute on Alcohol Abuse and Alcoholism, National Institutes of Health, Rockville, MD, USA

3 Division of General Internal Medicine and the Center for Interdisciplinary Research on AIDS, Yale University School of Medicine, New Haven, CT, USA

4 Department of Epidemiology of Microbial Diseases, Yale School of Public Health, Yale University, New Haven, CT, USA

5 Center for Health Equity Research and Promotion, Pittsburgh VA Healthcare System, Pittsburgh, PA, USA

6 Division of General Internal Medicine, Department of Medicine, Center for Research on Health Care, University of Pittsburgh, Pittsburgh, PA, USA

7 Veterans Aging Cohort Study Coordinating Center, Veterans Administration Connecticut Healthcare System, West Haven, CT, USA

8 VA Pittsburgh Healthcare System, Pittsburgh, PA, USA 